\title{
ChemComm
}

Cite this: Chem. Commun., 2014 , 50,4755

Received 7th February 2014, Accepted 21st March 2014

DOI: $10.1039 / \mathrm{c} 4 \mathrm{cc} 01014 \mathrm{k}$

www.rsc.org/chemcomm

\section{Water-soluble pyrrolopyrrole cyanine (PPCy) NIR fluorophores $\dagger$}

\author{
Simon Wiktorowski, Christelle Rosazza, Martin J. Winterhalder, Ewald Daltrozzo \\ and Andreas Zumbusch*
}

Water-soluble derivatives of pyrrolopyrrole cyanines (PPCys) have been synthesized by a post-synthetic modification route. In highly polar media, these dyes are excellent NIR fluorophores. Labeling experiments show how these novel dyes are internalized into mammalian cells.

Near-infrared (NIR) light absorbing and emitting compounds have attracted a lot of interest since the 1990's. ${ }^{1}$ Initially, this was motivated by their use in optical data storage or as laser dyes. Recently, however, new applications of NIR dyes have emerged, which has led to a surge of interest in the synthesis of such compounds. Examples for new applications are NIR chromophores as emitters in light-emitting diodes or as sensitizers in light-conversion materials, relevant in renewable energy related fields. ${ }^{2}$ In biomedical applications, NIR dyes are used as labels for fluorescence microscopy imaging of biomolecules and cellular structures. ${ }^{3}$ In addition, NIR fluorophores serve as contrast agents in cancer-targeted imaging, angiography and as sensitizers in photodynamic therapy. ${ }^{4}$ Here, NIR dyes offer several advantages over dyes absorbing and emitting in the UV/visible region: NIR light penetrates deeper into tissues due to lower scattering and lower absorption, natural autofluorescence of cellular components is lower and generally significantly reduced photodamage is observed in this spectral region. ${ }^{5}$ The use of NIR absorbing dyes is also facilitated by the ready availability of diode lasers as cheap excitation sources and the fact that Si-based detectors such as photodiodes and CCD cameras have their sensitivity maximum at around $800 \mathrm{~nm}^{6}$

In all of the aforementioned applications, NIR dyes with strong absorptions and emissions as well as with high photostability are desired. In order to be applicable in biological environments, the dyes need to be water-soluble. Therefore, many research efforts are currently dedicated to the development of new water-soluble NIR dyes or to the respective derivatization of NIR dyes from existing classes, like (naphthalo)cyanines, rylenes, squaraines, croconium

Fachbereich Chemie, Universität Konstanz, Universitätsstrasse 10, 78457 Konstanz, Germany. E-mail: andreas.zumbusch@uni-konstanz.de; Fax: +49 7531-88-3139

$\dagger$ Electronic supplementary information (ESI) available: Synthesis and characterisation of intermediates and final compounds. Additional spectroscopic data. Time-dependent changes in absorption spectra of final compounds in water. Data of photostability tests and intracellular emission spectra of $6^{\prime \prime}$. See DOI: 10.1039/c4cc01014k dyes, BODIPYs or others. ${ }^{7}$ Notable are also advances in other fields, like the engineering of GFP-related fluorescing proteins or quantum dots, which have resulted in the synthesis of novel systems with NIR emission. ${ }^{8}$ To date, however, only a few water-soluble dyes with strong NIR absorptions and emissions have been known. Apart from the general scarcity of NIR absorbing molecules, the main reason for this is that NIR absorption is commonly observed in extended $\pi$-systems which most often are hydrophobic. The incorporation of hydrophilic functionalities into hydrophobic dye structures, however, poses experimental difficulties. Additionally, even after the successful establishment of synthetic routes, one has to face problems with hydrolytic decomposition or dye aggregation, the latter often being accompanied by significant fluorescence quenching. ${ }^{7 d, 9}$ The currently most widely used water-soluble NIR dyes are heptamethine dyes like indocyanine green (ICG, cardio green), Cy7 or more stable and long-wavelength shifted derivatives thereof, which have been synthesized in recent years. ${ }^{3 f, 10}$ ICG is the only NIR fluorophore which has been approved by the U.S. Food and Drug Administration for medical use. ${ }^{3 e}$

Pyrrolopyrrole cyanines (PPCys) are a class of NIR dyes which has been introduced recently. ${ }^{11}$ PPCy chromophores exhibit narrow-band absorption and strong emission in the NIR region up to almost $1 \mu \mathrm{m}$. In general they are very photostable. ${ }^{12}$ The optical features and the structure-property relationships of PPCy dyes have been investigated in detail. ${ }^{13}$ They have proven to be attractive candidates for labeling applications, fluorescence lifetime imaging in vivo or as photoacoustic probes. ${ }^{14}$ While very recently, PPCy derivatives with highly polar structures have been reported, ${ }^{15}$ to date, however, no water-soluble derivatives of PPCys have been synthesized. In this contribution, we present a synthetic strategy to the development of water-soluble PPCys and discuss their optical properties. Live-cell imaging experiments are presented which demonstrate great potential of these compounds in imaging applications.

The preparation of water-soluble PPCys faces the problem that the hydrophobic core of the dyes needs to be functionalized with polar groups which mediate the solubility in polar solvents. Functionalities of this type, e.g. sulfonates, can generally be introduced either during the synthesis of the core chromophore structure or as a post-synthetic modification. The established routes for PPCy synthesis ${ }^{11-14}$ lead to 
specific problems which have to be solved. Since the use of e.g. alcohols as polar solvents for polar reactants is precluded by the use of phosphoryl chloride as an activator, we decided to introduce the polar groups via the modification of a suitably functionalized PPCy. However, also the introduction of suitable functionalities to which water-soluble groups can be attached after PPCy synthesis poses problems because PPCys are synthesized in a strongly acidic reaction from diketopyrrolopyrroles (DPPs), which themselves are prepared under strongly basic reaction conditions. Possible protective group strategies are hampered by the pigment character of DPPs, i.e. their low solubility in common organic solvents. Moreover, we found that the electrophilic character of phosphoryl chloride prevents conversion to PPCys if the reactants contain nucleophilic functional groups such as amines. A post-synthetic Pd-catalyzed alkyne substitution of aryl bromide functions in PPCys ( $c f$. compounds $\mathbf{3 b} \mathbf{b} \mathbf{4} \mathbf{b}$ from ref. 13a) also fails, most likely due to the insufficient solubility of these compounds. We therefore chose to synthesize a new DPP with terminal double bonds which can be converted to a PPCy before sulfonate groups are attached via a linker.

The synthetic scheme for the generation of water-soluble PPCys is shown in Scheme 1 and follows the general established route for PPCys described by our group up to $\mathrm{BPh}_{2}$-PPCys 4 ( $c f$. experimental section in the ESI $\dagger) .{ }^{12 a}$ DPP 1 is allowed to react with two equivalents of 2-(6-tert-butylbenzothiazol-2-yl)-acetonitrile $2^{\prime}$ or 2-(6-tert-butylquinoline-2-yl)acetonitrile $2^{\prime \prime}$, respectively, by activation through phosphoryl chloride to yield the hydrogen chelates $3^{\prime}$ or $3^{\prime \prime}$, respectively. These H-PPCys $\mathbf{3}$ are converted to the benzothiazole substituted $\mathrm{BPh}_{2}-\mathrm{PPCy} \mathbf{4}^{\prime}$ or quinoline substituted $\mathrm{BPh}_{2}-\mathrm{PPCy}$ $\mathbf{4}^{\prime \prime}$, respectively. Subsequent radical addition of mercaptoacetic acid to the four terminal double bonds yields the carboxylated $\mathrm{BPh}_{2}$-PPCys $5^{\prime}$ and $5^{\prime \prime}$. In the final step, the carboxylated compounds are converted to the water-soluble derivatives $\mathbf{6}^{\prime}$ and $\mathbf{6}^{\prime \prime}$ by NHS activation of the four carboxylic acid functions and subsequent fourfold amidation through the sodium salt of 2-aminoethanesulfonic acid.

Fig. 1 (top) shows the normalized absorption spectra of benzothiazole substituted $\mathrm{BPh}_{2}$-PPCy $\mathbf{6}^{\prime}$ and quinoline substituted $\mathrm{BPh}_{2}-\mathrm{PPCy}$ $6^{\prime \prime}$ in water with absorption maxima at around $800 \mathrm{~nm}$. The solubility of both compounds in pure water is in the $\mathrm{mM}$ range. When dissolving these compounds in water the absorption spectra shift very slightly over a period of hours ( $c f$. Fig. S1 in ESI $\dagger$ ). These changes in absorbance for the pure aqueous milieu are more pronounced in the case of $6^{\prime \prime}$ compared to $\mathbf{6}^{\prime}$. We assign them to conformational rearrangements of the long alkyl chains and solvation effects. As is the case for other PPCys dissolved in nonpolar solvents, the half widths of the absorption and emission bands of these compounds are also very small in polar solvents $\left(\Delta \tilde{\nu}_{1 / 2}^{\mathrm{A}}=650(575) \mathrm{cm}^{-1}\right.$ for $6^{\prime}$ and $859(539) \mathrm{cm}^{-1}$ for $6^{\prime \prime}$ in water (methanol)). We do not observe any bands which could be attributed to dye aggregation. Therefore we conclude that if any aggregation occurs in these systems, the respective chromophore interactions must be very weak. A possible explanation for the absence of aggregation is the steric demand of both the $\mathrm{BPh}_{2}$ groups and the long alkyl chains, which hinder $\pi-\pi$-interactions. For this reason the complexing agent $\mathrm{BPh}_{2} \mathrm{Cl}$ was preferably chosen over $\mathrm{BF}_{3} \cdot \mathrm{OEt}_{2}$, which was also frequently used in order to obtain strongly fluorescent PPCys. The fluorescence quantum yields for $6^{\prime}$ and $6^{\prime \prime}$ range between 1 and $34 \%$ in water, methanol and DMSO, respectively ( $c f$. Table 1; for supplemented spectroscopic data see Table S1, ESI $\dagger$ ). Since there is no evidence for dye-dye aggregates from the absorption spectra, we attribute the reduction of fluorescence quantum yields in water to solvation effects rather than to dye aggregation. Reduced quantum yields in water are often found for different dyes, as exemplarily shown
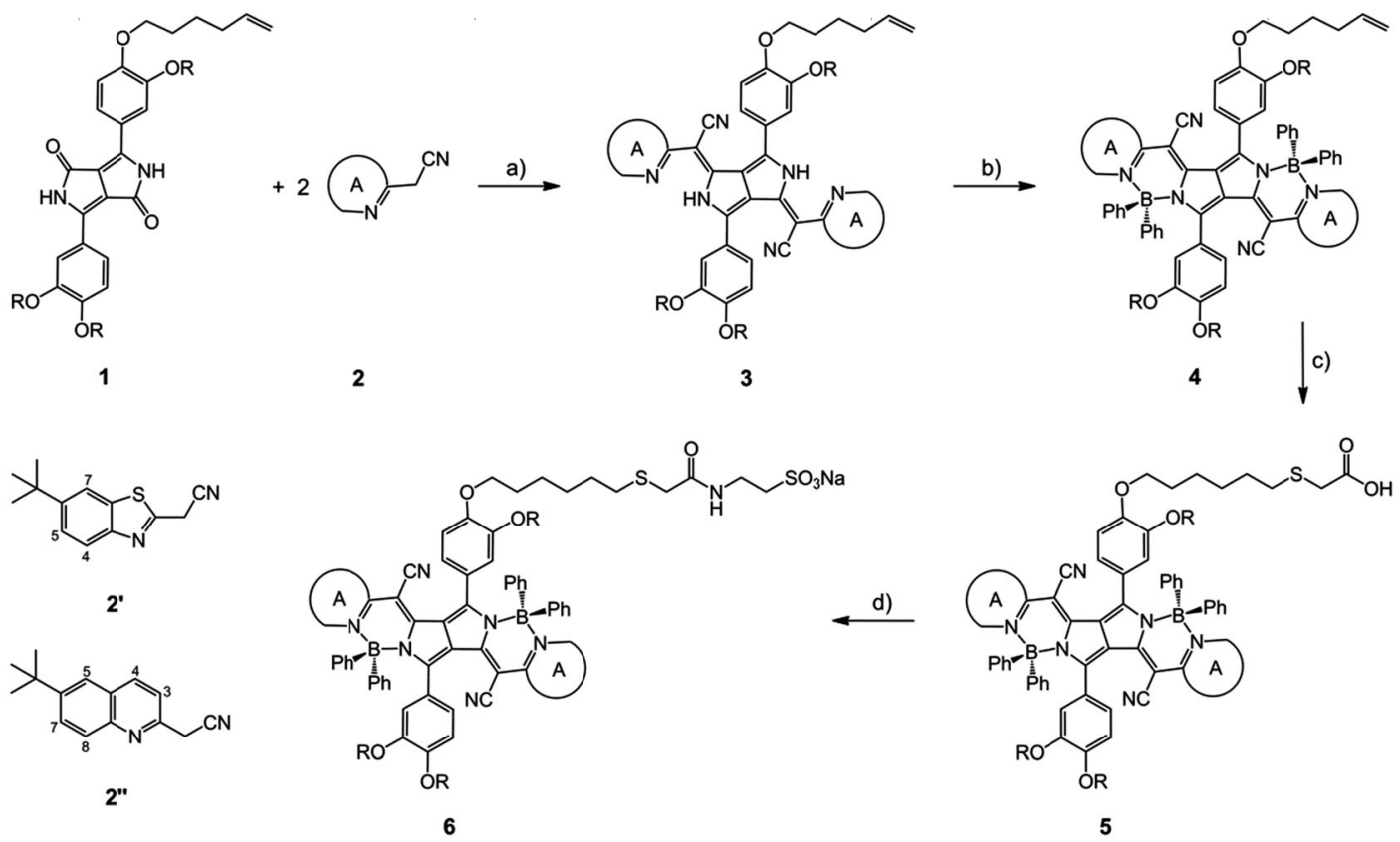

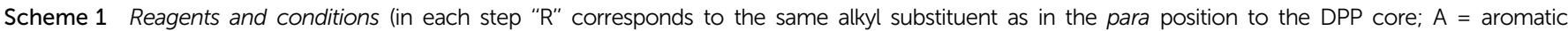

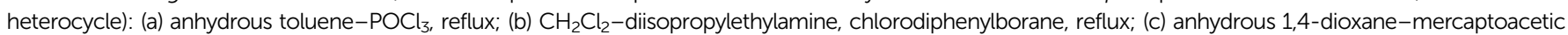
acid, AIBN, $90{ }^{\circ} \mathrm{C}$ or reflux. (d) (1) $\mathrm{CH}_{2} \mathrm{Cl}_{2}-\mathrm{DCC}-\mathrm{NHS}$, room temperature; (2) DMF, 2-aminoethanesulfonic acid sodium salt, room temperature. 

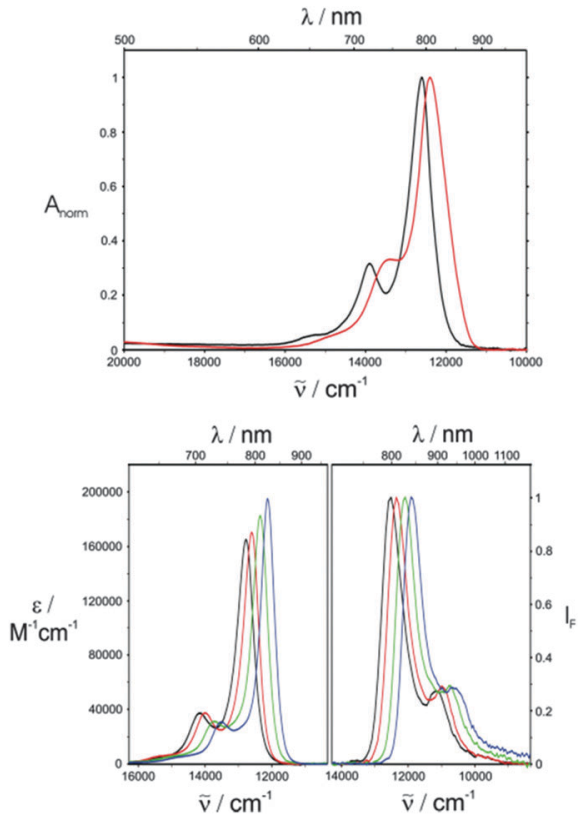

Fig. 1 (top) Normalized absorption spectra of benzothiazole substituted $\mathrm{BPh}_{2}$-PPCy $\mathbf{6}^{\prime}$ (black) and quinoline substituted $\mathrm{BPh}_{2}$-PPCy $\mathbf{6}^{\prime \prime}$ (red) in water. (bottom) Absorption spectra (left) and normalized fluorescence spectra (right) of benzothiazole substituted $\mathrm{BPh}_{2}-\mathrm{PPCy} \mathbf{6}^{\prime}$ in methanol (black) and DMSO (red) and quinoline substituted $\mathrm{BPh}_{2}-\mathrm{PPCy} \mathbf{6}^{\prime \prime}$ in methanol (green) and DMSO (blue).

for ICG (Table 1). In all solvents, the emission maxima are at or slightly above $800 \mathrm{~nm}$ ( $c$. Fig. 1 (bottom) and Table 1). Two important parameters for imaging applications are the optical brightness, defined in different publications as the product of the extinction coefficient of the absorption maximum $\varepsilon_{\max }$ and the fluorescence quantum yield $\Phi_{\mathrm{F}}{ }^{16}$ and the photostability of the dyes. As shown in Table 1, the brightness of $\mathbf{6}^{\prime}$ and $\mathbf{6}^{\prime \prime}$ is high in polar organic solvents with values of 33000 to $66000 \mathrm{M}^{-1} \mathrm{~cm}^{-1}$ compared to ICG in methanol with a value of $16000 \mathrm{M}^{-1} \mathrm{~cm}^{-1}$.

Concerning the photostability, we have shown earlier that PPCys exhibit very little photobleaching in organic solvents like chloroform. ${ }^{12 a}$ In order to test the photostability in water, a cuvette containing an aqueous solution of $6^{\prime \prime}$ was exposed to daylight for several hours. Solutions of ICG in water and of compound 10e from ref. $12 a$ in chloroform were exposed to the same illumination conditions. These experiments show that while $\mathrm{BPh}_{2}-\mathrm{PPCy} 6^{\prime \prime}$ is less photostable in water than 10e in chloroform, it is much more photostable than ICG in water ( $c f$. Fig. S2 in ESI $\dagger$ ). The main advantage of ICG is its low toxicity. While no data on the toxicity of PPCys are available, one can note that several days after injection of PPCy solutions, no adverse effects on mice were observed. ${ }^{14 b}$ Moreover, DPPs, the core structure of PPCys, have been shown to be non-toxic by either oral or dermal uptake and show no mutagenic effects in vitro and in vivo. ${ }^{18}$

As pointed out before, water-soluble NIR fluorophores have great potential in biomedical applications. In order to evaluate the suitability of the new water-soluble $\mathrm{BPh}_{2}$-PPCys as fluorescence probes, live-cell images of $\mathrm{CHO}$ cells incubated with $\mathbf{6}^{\prime \prime}$ were taken using a confocal fluorescence microscope. Incubation was performed with a $1 \mu \mathrm{M}$ solution of $6^{\prime \prime}$ in cell culture medium for 30 minutes. No additional solvents or surfactants had to be used since $\mathbf{6}^{\prime \prime}$ is well soluble in
Table 1 Spectroscopic data for $\mathrm{BPh}_{2}$-PPCys $\mathbf{6}^{\prime}$ and $\mathbf{6}^{\prime \prime}$ in different solvents and in $\mathrm{CHO}$ cells compared to ICG. $\lambda_{\text {max }}^{\mathrm{A}}$ : wavelength of the absorption maximum, $\lambda_{\text {max }}^{\mathrm{F}}$ : wavelength of the fluorescence maximum, $\varepsilon_{\max }$ : molar decadic absorption coefficient of the absorption maximum, $\Phi_{\mathrm{F}}$ : fluorescence quantum yield, $\varepsilon_{\max } \Phi_{\mathrm{F}}$ : optical brightness

\begin{tabular}{|c|c|c|c|c|c|c|}
\hline & & $\begin{array}{l}\lambda_{\max }^{\mathrm{A}} \\
{[\mathrm{nm}]}\end{array}$ & $\begin{array}{l}\lambda_{\max }^{\mathrm{F}} \\
{[\mathrm{nm}]}\end{array}$ & $\begin{array}{l}\varepsilon_{\max }{ }^{a} \\
{\left[\mathbf{M}^{-1} \mathrm{~cm}^{-1}\right]}\end{array}$ & $\Phi_{\mathrm{F}}$ & $\begin{array}{l}\varepsilon_{\max } \Phi_{\mathrm{F}} \\
{\left[\mathrm{M}^{-1} \mathrm{~cm}^{-1}\right]}\end{array}$ \\
\hline \multirow[t]{3}{*}{$6^{\prime}$} & DMSO & 794 & 805 & 171000 & 0.27 & 46000 \\
\hline & $\mathrm{MeOH}$ & 782 & 800 & 166000 & 0.20 & 33000 \\
\hline & $\mathrm{H}_{2} \mathrm{O}$ & 793 & 800 & 125000 & 0.01 & 1000 \\
\hline \multirow[t]{4}{*}{$6^{\prime \prime}$} & DMSO & 825 & 840 & 195000 & 0.34 & 66000 \\
\hline & $\mathrm{MeOH}$ & 810 & 825 & 183000 & 0.25 & 46000 \\
\hline & $\mathrm{H}_{2} \mathrm{O}$ & $805^{b}$ & 825 & 110000 & 0.01 & 1000 \\
\hline & CHO cell & $-^{c}$ & 840 & $-^{c}$ & 0.34 & $-^{c}$ \\
\hline \multirow[t]{2}{*}{ ICG } & $\mathrm{MeOH}^{16 b}$ & 785 & 807 & 204000 & 0.08 & 16000 \\
\hline & $\mathrm{H}_{2} \mathrm{O}^{17}$ & 775 & 820 & 155000 & 0.003 & 500 \\
\hline
\end{tabular}

${ }^{a}$ Values corrected for ten and three molecules of water per formula unit for $\mathbf{6}^{\prime}$ and $6^{\prime \prime}$, respectively ( $c f$. general remarks in the experimental section, ESI). ${ }^{b}$ Can range from 805 to $820 \mathrm{~nm}$ (conformation/solvation effect; see text). ${ }^{c}$ Not determined.

aqueous solution. For the microscopy experiments, we employed a scanning stage confocal microscope which allowed excitation with $690 \mathrm{~nm}$ light as the longest available wavelength. Despite the fact that this wavelength is far off the absorption maximum of $6^{\prime \prime}$ such that it has only a low extinction at this wavelength (see Fig. 1) and the fact that low excitation powers were used $(\sim 5 \mu \mathrm{W})$, a strong fluorescence signal was readily obtained. Fig. 2 (left) shows a high concentration of dye $6^{\prime \prime}$ in vesicular structures in the cytoplasm and low concentrations in the cell plasma membrane. The data show that $\mathrm{BPh}_{2}$-PPCy $\mathbf{6}^{\prime \prime}$ enters the cells without application of any permeability-enhancing techniques like e.g. electroporation. This is surprising because the molecular weight of the dye is comparably high $(\sim 2.3 \mathrm{kDa})$. Together with its overall negative charge, this should make direct diffusion through the plasma membrane less probable. In order to learn more about the entry process, especially whether the dye enters by endocytosis or by transmembrane diffusion, and about the compartments it ends up in the cells, incubation experiments were performed at different temperatures. Since endocytotic processes are strongly suppressed at $4{ }^{\circ} \mathrm{C}$, one expects to find a very low dye concentration in the cytoplasm upon incubation at this temperature if endocytosis is the main entering pathway. Several incubations of $\mathrm{CHO}$ cells were performed at $4{ }^{\circ} \mathrm{C}$ along with control experiments at $37^{\circ} \mathrm{C}$ using a $100 \mu \mathrm{M}$ solution of $6^{\prime \prime}$ in cell culture medium for 30 minutes. Confocal fluorescence images of fixed cells were taken in a z-scanning mode (see maximum projections in Fig. 2 (middle) for incubation at $4{ }^{\circ} \mathrm{C}$ and Fig. 2 (right) for incubation at $37^{\circ} \mathrm{C}$; excitation powers $<1 \mu \mathrm{W}$ ). Cells incubated at $4{ }^{\circ} \mathrm{C}$ indeed show only very weak, diffuse fluorescence emission and do not feature the vesicular dye structures observed in cells incubated at $37{ }^{\circ} \mathrm{C}$, even though a very high dye concentration of $100 \mu \mathrm{M}$ was used. This result points at endocytosis rather than diffusion through the plasma membrane being the main pathway for cellular uptake of $6^{\prime \prime}$. It also means that the observed structures are most likely endosomes.

Because emission of $6^{\prime \prime}$ in CHO cells seemed to be fairly strong, we were interested in determining the intracellular quantum yield of this dye. For this purpose, first emission spectra of the vesicular structures of a series of CHO cells incubated with $6^{\prime \prime}$ were recorded ( $c f$. Fig. S3 in ESI $\dagger$ ). It was found that the fluorescence spectrum matches that of $6^{\prime \prime}$ in a DMSO solution, indicating that emission of 

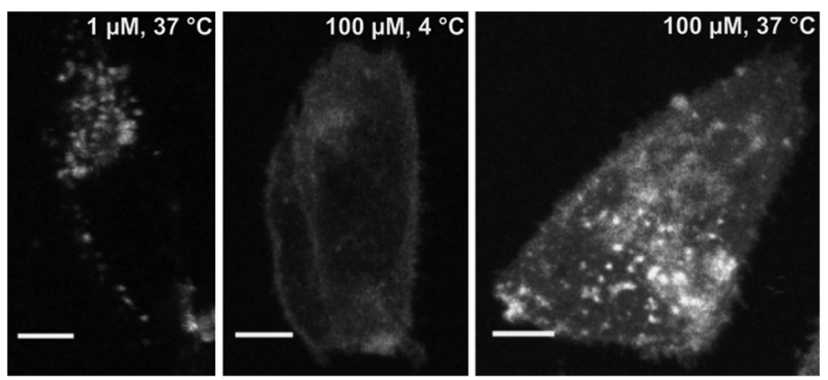

Fig. 2 Confocal fluorescence images of $\mathrm{CHO}$ cells incubated with $\mathbf{6}^{\prime \prime}$ taken within about one hour after incubation for 30 minutes (excitation wavelength: $690 \mathrm{~nm}$, detection wavelength: $>770 \mathrm{~nm}$; see ESI†); scalebars: $5 \mu \mathrm{m}$.

$6^{\prime \prime}$ originates from the same form of the dye as in solution. Subsequently, several fluorescence lifetime images of the same cells were taken and evaluated ( $c f$. Fig. S3 in ESI $\dagger$ ). The average fluorescence lifetime of $\mathbf{6}^{\prime \prime}$ in these cells was found to be $2.5 \mathrm{~ns}$. The fluorescence lifetime of $6^{\prime \prime}$ was also determined in solution for different solvents and found to be $2.47 \mathrm{~ns}$ in DMSO. As the intracellular emission spectrum of $6^{\prime \prime}$ matches that obtained from the DMSO solution, it indicates that the radiative rate constant $k_{\mathbf{F}}$ has the same magnitude in both cases. As a consequence, the intracellular fluorescence quantum yield is the same as that obtained in the DMSO solution. We therefore conclude that the intracellular fluorescence quantum yield of $6^{\prime \prime}$ is $34 \%$ (Table 1). The fact that these quantum yields are much higher than those observed in aqueous solution most likely is due to uptake into a relatively hydrophobic environment.

In conclusion, we report the synthesis of two novel pyrrolopyrrole cyanine dyes as the first water-soluble representatives of this class of NIR fluorophores. The synthesis followed a post-synthetic functionalization route. The new PPCy dyes thus obtained exhibit good solubility in pure water ( $\mathrm{mM}$ range). The half widths of the absorption spectra in water are very low and no distinct spectral bands attributable to dye-dye aggregates were found. Quantum yields and extinction coefficients are high in polar organic solvents like DMSO and methanol resulting in a high optical brightness of up to $66000 \mathrm{M}^{-1} \mathrm{~cm}^{-1}$. The photostability of the new dyes in water was tested for $\mathbf{6}^{\prime \prime}$ and found to be significantly higher than that of ICG. Confocal fluorescence imaging was performed on $\mathrm{CHO}$ cells incubated with $\mathbf{6}^{\prime \prime}$. Experimental evidence indicates that the dyes are internalized by endocytotic processes. The intracellular quantum yield of $6^{\prime \prime}$ was determined to be $34 \%$ by evaluation of fluorescence lifetime imaging data. All of these results in summary show that the new water-soluble PPCy dyes hold great promise for imaging applications both on a cellular and on a tissue level.

Financial support by the DFG, SFB 767, TP B3 is acknowledged.

\section{Notes and references}

1 (a) J. Fabian and R. Zahradnik, Angew. Chem., Int. Ed. Engl., 1989, 28, 677; (b) J. Fabian, J. Prakt. Chem., 1991, 333, 197; (c) J. Fabian, H. Nakazumi and M. Matsuoka, Chem. Rev., 1992, 92, 1197.

2 (a) G. Qian and Z. Y. Wang, Chem. - Asian J., 2010, 5, 1006; (b) U. Mayerhöffer, K. Deing, K. Gruß, H. Braunschweig, K. Meerholz and F. Würthner, Angew. Chem., Int. Ed., 2009, 48, 8776.

3 (a) J. O. Escobedo, O. Rusin, S. Lim and R. M. Strongin, Curr. Opin. Chem. Biol., 2010, 14, 64; (b) S. A. Hilderbrand and R. Weissleder, Curr. Opin. Chem. Biol., 2010, 14, 71; (c) A. Becker, C. Hessenius, K. Licha, B. Ebert, U. Sukowski, W. Semmler, B. Wiedenmann and
C. Grötzinger, Nat. Biotechnol., 2001, 19, 327; (d) M. Y. Berezin and S. Achilefu, Chem. Rev., 2010, 110, 2641; (e) H. Kobayashi, M. Ogawa, R. Alford, P. L. Choyke and Y. Urano, Chem. Rev., 2010, 110, 2620; $(f)$ E. M. Sevick-Muraca, J. P. Houston and M. Gurfinkel, Curr. Opin. Chem. Biol., 2002, 6, 642; $(g)$ A. Loudet, R. Bandichhor, K. Burgess, A. Palma, S. O. McDonnell, M. J. Hall and D. F. O'Shea, Org. Lett., 2008, 10, 4771.

4 (a) S. L. Luo, E. L. Zhang, Y. P. Su, T. M. Cheng and C. M. Shi, Biomaterials, 2011, 32, 7127; (b) M. Wainwright, Color. Technol., 2010, 126, 115; (c) B. Riefke, K. Licha and W. Semmler, Radiologe, 1997, 37, 749; (d) B. Ballou, L. A. Ernst and A. S. Waggoner, Curr. Med. Chem., 2005, 12, 795; (e) A. Yuan, J. H. Wu, X. L. Tang, L. L. Zhao, F. Xu and Y. Q. Hu, J. Pharm. Sci., 2013, 102, 6; $(f)$ R. Weissleder, C. H. Tung, U. Mahmood and A. Bogdanov, Nat. Biotechnol., 1999, 17, 375.

5 R. Weissleder, Nat. Biotechnol., 2001, 19, 316.

6 H. W. Siesler, Y. Ozaki, S. Kawata and H. M. Heise, Near-infrared spectroscopy: principles, instruments, applications, Wiley- $\mathrm{VCH}$, Weinheim, 2002.

7 (a) N. C. Yates, J. Moan and A. Western, J. Photochem. Photobiol., B, 1990, 4, 379; (b) J. D. Spikes, J. E. Vanlier and J. C. Bommer, J. Photochem. Photobiol., C, 1995, 91, 193; (c) C. Jung, N. Ruthardt, R. Lewis, J. Michaelis, B. Sodeik, F. Nolde, K. Peneva, K. Mullen and C. Brauchle, ChemPhysChem, 2009, 10, 180; (d) E. L. Cole, E. Arunkumar, S. Z. Xiao, B. A. Smith and B. D. Smith, Org. Biomol. Chem., 2012, 10, 5769; (e) X. Z. Song and J. W. Foley, Dyes Pigm., 2008, 78, 60; $(f)$ Y. J. Yang, M. Lowry, X. Y. Xu, J. O. Escobedo, M. Sibrian-Vazcluez, L. Wong, C. M. Schowalter, T. J. Jensen, F. R. Fronczek, I. M. Warner and R. M. Strongin, Proc. Natl. Acad. Sci. U. S. A., 2008, 105, 8829; (g) S. L. Zhu, N. Dorh, J. T. Zhang, G. Vegesna, H. H. Li, F. T. Luo, A. Tiwari and H. Y. Liu, J. Mater. Chem., 2012, 22, 2781; (h) K. Umezawa, D. Citterio and K. Suzuki, Anal. Sci., 2008, 24, 213.

8 (a) G. S. Filonov, K. D. Piatkevich, L. M. Ting, J. H. Zhang, K. Kim and V. V. Verkhusha, Nat. Biotechnol., 2011, 29, 757; (b) X. Michalet, F. F. Pinaud, L. A. Bentolila, J. M. Tsay, S. Doose, J. J. Li, G. Sundaresan, A. M. Wu, S. S. Gambhir and S. Weiss, Science, $2005,307,538$.

9 (a) F. Biedermann, E. Elmalem, I. Ghosh, W. M. Nau and O. A. Scherman, Angew. Chem., Int. Ed., 2012, 51, 7739; (b) H. Langhals, R. Ismael and O. Yuruk, Tetrahedron, 2000, 56, 5435.

10 (a) W. Pham, W. F. Lai, R. Weissleder and C. H. Tung, Bioconjugate Chem., 2003, 14, 1048; (b) W. Pham, Z. Medarova and A. Moore, Bioconjugate Chem., 2005, 16, 735; (c) W. Pham, L. Cassell, A. Gillman, D. Koktysh and J. C. Gore, Chem. Commun., 2008, 1895; (d) H. S. Choi, K. Nasr, S. Alyabyev, D. Feith, J. H. Lee, S. H. Kim, Y. Ashitate, H. Hyun, G. Patonay, L. Strekowski, M. Henary and J. V. Frangioni, Angew. Chem., Int. Ed., 2011, 50, 6258; (e) S. A. Hilderbrand, K. A. Kelly, R. Weissleder and C. H. Tung, Bioconjugate Chem., 2005, 16, 1275.

11 G. M. Fischer, A. P. Ehlers, A. Zumbusch and E. Daltrozzo, Angew. Chem., Int. Ed., 2007, 46, 3750.

12 (a) G. M. Fischer, M. Isomäki-Krondahl, I. Göttker-Schnetmann, E. Daltrozzo and A. Zumbusch, Chem. - Eur. J., 2009, 15, 4857; (b) G. M. Fischer, E. Daltrozzo and A. Zumbusch, Angew. Chem., Int. Ed., 2011, 50, 1406.

13 (a) G. M. Fischer, M. K. Klein, A. Zumbusch and E. Daltrozzo, Eur. J. Org. Chem., 2011, 3421; (b) S. Wiktorowski, G. M. Fischer, M. J. Winterhalder, E. Daltrozzo and A. Zumbusch, Phys. Chem. Chem. Phys., 2012, 14, 2921.

14 (a) G. M. Fischer, C. Jüngst, M. Isomäki-Krondahl, D. Gauss, H. M. Möller, E. Daltrozzo and A. Zumbusch, Chem. Commun., 2010, 46, 5289; (b) M. Y. Berezin, W. J. Akers, K. Guo, G. M. Fischer, E. Daltrozzo, A. Zumbusch and S. Achilefu, Biophys. J., 2009, 97, L22; (c) M. Bai and S. Achilefu, Heterocycl. Commun., 2010, 16, 213.

15 M. Z. Zhou, X. Zhang, M. F. Bai, D. W. Shen, B. G. Xu, J. Kao, X. Ge and S. Achilefu, R. Soc. Chem. Adv., 2013, 3, 6756.

16 (a) N. C. Shaner, P. A. Steinbach and R. Y. Tsien, Nat. Methods, 2005, 2, 905; (b) H. Lee, J. C. Mason and S. Achilefu, J. Org. Chem., 2006, 71, 7862.

17 (a) R. C. Benson and H. A. Kues, Phys. Med. Biol., 1978, 23, 159; (b) O. G. Bjornsson, R. Murphy, V. S. Chadwick and S. Bjornsson, J. Clin. Chem. Clin. Biochem., 1983, 21, 453.

18 M. Zillgitt, Colourants for Food Contact Plastics, Neumann Druck, Heidelberg, Germany, 2002. 